\title{
Natural history of hip instability in infants (without subluxation or dislocation): a three year follow-up
}

\author{
Blazej Pruszczynski ${ }^{1}$, H Theodore Harcke ${ }^{2}$, Laurens Holmes $\mathrm{Jr}^{3}$ and J Richard Bowen ${ }^{4^{*}}$
}

\begin{abstract}
Background: The natural history of hip instability (without subluxation or dislocation) and treatment in infants remain controversial. We performed a retrospective cohort case-only study with blinded, prospectively collected data to assess normalization of the acetabular index in consecutive untreated infant hips with sonography instability.

Methods: Consecutive hips meeting inclusion criteria were followed by sonography/radiography and data analyzed using tabular and regression models.

Results: In 48 hips, acetabular index measured by radiography normalized within 3 years of age without treatment. Normalization by age occurred: 7 months in 35\%, 12 months in 67\%, 18 months in 75\%, 24 months in 81\%, and 36 months in 100\%. Two patterns of normalization of the acetabular index were observed: group I showed ossification in a physiological range of normal by 7 months of age, and group II had delayed ossification with later normalization of the acetabular index measurement. Breech presentation ( $p=0.013)$ and cesarean delivery $(p=0.004)$ statistically directly correlated with a later normalization.

Conclusions: The natural history of infant hip instability (without subluxation or dislocation), which is reduced at rest and unstable with stress as diagnosed by the Harcke method of sonography, has spontaneous normalization of the acetabular index within 3 years of age. We suggest three patterns of acetabular ossification in unstable infants' hips: (I) normal ossification, (II) delayed ossification with normalization of the acetabular index by age 3 years, and (III) defective secondary centers of ossification with an upward tilt of the lateral acetabular rim in adolescence.
\end{abstract}

Keywords: DDH, Hip instability, Hip treatment, Hip dysplasia, Natural history

\section{Background}

Developmental dysplasia of the hip (DDH) is an abnormality of the hip with pathologic alteration in size, shape, and organization of cells [1] and is manifested by both soft tissue (stability) and bony (acetabular) components. Prior to sonography, the infant hip was assessed clinically and confirmed radiographically. Sonography by the Harcke method evaluates the hip joint for soft tissue stability and bone and cartilage abnormality, which are reported as 1) position (being either reduced, subluxated, or dislocated), 2) stability (normal, lax, dislocatable, reducible, or not reducible), and 3) dysplasia

\footnotetext{
* Correspondence: Richard.Bowen@nemours.org

${ }^{4}$ Department of Orthopedics, Nemours/Alfred I. duPont Hospital for Children, 1600 Rockland Rd, Wilmington, DE 19803, USA

Full list of author information is available at the end of the article
}

(measured as percent of head coverage or acetabular bone inclination, as with the Graf $\alpha$ angle) [2-5]. The natural history of infant hips manifesting instability with stress, which are reduced at rest and which may or may not have acetabular dysplasia by sonography, is not clear, and this evokes controversy regarding management [2-17].

The aim of our study was to observe a consecutive group of infants who met the criteria of hip instability with stress, whose hips are reduced at rest and which may or may not have acetabular dysplasia by sonography (measured as percent of head coverage or acetabular bone inclination as with the Graf $\alpha$ angle), to determine the natural history of hip instability without treatment. We hypothesize that the acetabular index (AI) in these hips will normalize. 


\section{Methods}

With Institutional Review Board (Nemours Institutional Review Board, Jacksonville, FL) approval, we conducted a retrospective cohort case-only study on prospectively collected data involving the natural history of infant hips with specific sonographic findings: joint instability under stress but reduced at rest that may or may not have acetabular dysplasia by sonography (measured as percent of head coverage or acetabular bone inclination, as with the Graf $\alpha$ angle). These infants were followed by the same treating surgeon, and the data were "blindly" reviewed by a non-treating author and analyzed by a biostatistician author. The treating surgeon followed each patient with imaging using the center edge angle as a reference of resolving dysplasia.

The inclusion criteria consisted of the following: 1) consecutive cases with risk of typical DDH (no syndromic conditions), 2) infants younger than two months at first sonography, 3) sonography by the Harcke method showing hips that were reduced at rest and unstable with stress, and 4) radiographic follow up until the normalization of the AI. Swaddling was not allowed.

The exclusion criteria were hips that were dislocated (demonstrated by Ortolani positive test) or dislocatable (manifested by Barlow positive test) [18,19]. Demographic data included sex, ages at imaging, race, delivery presentation/type of delivery, torticollis, and family history of DDH.

Dynamic sonography was performed utilizing a 12.5 MHz linear transducer in infants up to 6 months of age. Criteria for quality images included: 1) horizontal iliac line, 2) visible acetabular roof (ilium and pubis), and 3) posterior acetabulum (ischium) according to the Harcke technique $[4,5]$. Measurements were made on coronal neutral images, coronal flexion images (with and without stress), and transverse flexion images (with and without stress). These measurements were repeated for first (FS) and last (LS) sonographies at time periods we considered critical for statistical analysis. The $\alpha$ angle and sonographical central edge angle ( $\mathrm{sCE}$ ) were measured only in coronal views. To ensure standardization of sonographic images, the rule of $1 \mathrm{~mm}$ accepted difference in the measurements of the acetabulum and the diameter of the femoral head was established for neutral and adduction views of the same hip. The list of measured values is presented in Additional file 1.

Acetabular index and the center-edge angle of Wiberg (CE) were measured (following Tönnis's description) on the first anteroposterior radiographs for baseline and again on the radiograph showing normalization of the AI $[20,21]$. The value for normalization of the AI was derived from the data of Tönnis and Caffey, and blended statistical analysis revealed $\leq 25^{\circ} \mathrm{AI}$ as normative $[20,21]$.
The age in which the hip achieved an $\mathrm{AI} \leq 25^{\circ}$ was considered the "age of acetabular index normalization."

\section{Sample size and power estimations}

Statistical issues considered included sample size, power estimations, and data analysis. The 26 patients who met the inclusion criteria (consecutive sampling) provided data on 48 hips, reflecting the study size. To estimate the statistical power of the study, we used " $\alpha$ " (type one error tolerance for $5 \%$ ), effect size of $10 \%(0.1)$, and repeated measure design implying $\mathrm{AI}$ at the first radiography taken (mean $=28.0, \mathrm{SD}=4.0$ ) and the radiography with "normalization of AI" value (mean $=19.8, \mathrm{SD}=3.8$ ). With these parameters, we estimated the power to be sufficient $(>80 \%)$ in determining the $10 \%$ change in AI comparing initial radiography and radiography with normalization of the AI.

\section{Statistical analysis}

The discrete and categorical data were summarized with frequency and percentages. A normality test was performed to examine continuous data for shape, spread, and distribution. The summary statistic for the normally distributed data was achieved with the mean and standard deviation (SD), while data that violated the normality assumption were summarized with median and interquartile range (IQR). Chi-squared statistic and Fisher's exact test were used to examine the distribution of categorical variables by the time of normalization of the AI. When the expected cell count was $<2.0$, Fisher's exact test was used to adjust for the small expected cell count. To examine the predictors of AI normalization, we used a univariable unconditional logistic regression model. This model is adequate in examining predictors of a given outcome if the outcome is measured on a binary scale. The binary scale for our outcome variable (normalization of AI) was derived from the continuous variable by using cut-off points that define normal vs. late normalization. Further, we performed a multivariable logistic regression model by using a forward loading and backward elimination approach and adjusted the significance level to the numbers of variables introduced into the model following Bonferroni suggestions $(0.05 / \mathrm{n})$, where " $\mathrm{n}$ " is the number of variables entered into the multivariable model building. The rationale for the Bonferroni suggestion is to adjust for multiple comparisons in the model, which, if not addressed, will introduce measurement error into the inference.

Prior to the analysis, to determine the natural history of AI normalization, a paired sample $t$-test was used for normally distributed data; otherwise, the Wilcoxon rank-sum test was used. To examine the factors that differentiated the children with normalization of the 
AI at different months of age [5-12], we used repeated measures analysis of variance (RANOVA).

All tests were two-tailed, and the significance level for univariable analysis was set at $<0.05$. STATA statistical software, version 12.0 (STATA Corp., College Station, TX), was used to perform all the analyses.

An additional insightful case (that was not part of the natural history samples) is provided to enhance discussion of our results. This 12-year-old girl presented after falling, and radiographs showed flattening of the lateral acetabular rim and delayed ossification of the secondary ossification centers of the acetabulum; the $\mathrm{AI}<25^{\circ}$ was bilateral (Figure 1). In early infancy, she was evaluated by our institution with Harcke method sonography, which showed the hip reduced at rest and unstable with stress. She was born at term by vaginal delivery without history of breech presentation. Radiographs at 5 years of age showed a normal AI. Currently, she is still asymptomatic; however, we are concerned about the potential for arthritis in adulthood from the flattening of the lateral acetabular rim. Additionally, the senior author treated this patient's younger sister with bilateral dislocated hips in a Pavlik harness.

\section{Results}

This cohort case-only study consisted of 48 hips (25 patients). Sonography of one hip each in two patients did not meet inclusion criteria. No patient requested a change in treatment. Twenty-one patients were Caucasian (84\%), one Asian (4\%), and one Native American (4\%), and, in two cases, race was undeclared (8\%). The median age at the first sonography was 4 weeks (range 1-8 weeks);

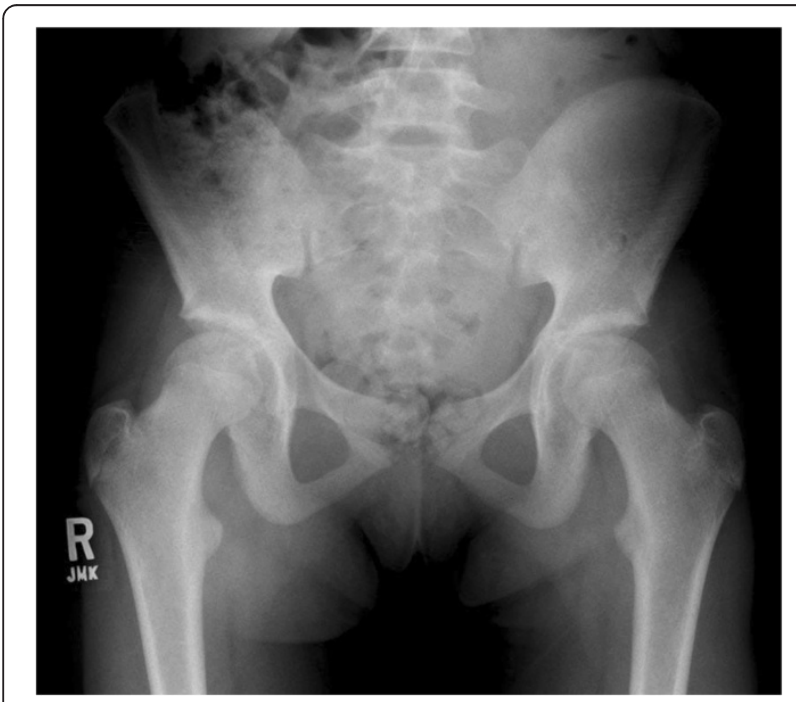

Figure 1 Anteroposterior radiograph of the pelvis of the additional insightful patient at age $\mathbf{1 2}$ years. The outward margin of the right acetabulum is tilted upward, and no secondary centers of ossification are present in the hip. the second sonography was performed at a median of 8 weeks (range 8-24 weeks). The first radiograph was performed at a mean age of 21 weeks $(\mathrm{SD}=6.4$, range 12-36 weeks). The radiograph with normalized AI was performed at a median of 42 weeks of age (range 12-228 weeks). Thirty-nine hips were from girls $(81.2 \%)$, and a family history of DDH was observed in 4 hips (8.3\%). A total of 27 hips were breech (56.2\%), 1 was transverse (2.1\%), and 2 had missing data (4.2\%). The type of delivery was cesarean section in 32 hips (66.7\%).

Analysis of clinical and sonographic factors established two different groups of normalization of the AI of eligible infant hips: before 7 months of age (group I), and after 7 months of age (group II) (Table 1). Analysis of sonographic data concluded that three values were statistically significant (LSCNmid, LSCFaddMAD, LSCFmad) and that three values were almost significant (LSCFaddBC, LSCNmad, FSCF $\Delta$ sce). Subsequent analysis of difference between groups I and II showed four sonographic factors that were statistically significant (LSCNmid, LSCFmad, LSCFaddBC, LSCFaddMID) and three factors were almost significant (FFSCF $\Delta$ sce, FSTF $\Delta \mathrm{a} / \Delta$ d, LSTFmad) (Table 2). A combination of sonographic and clinical factors indicated the best cutoff time between groups to be at or before 7 months of age. Breech presentation $(p=0.013)$ and cesarean delivery $(p=0.004)$ statistically correlated with a slower normalization of AI (group II) (Table 3).

Since some orthopedists use the Graf $\alpha$ angle, some use $50 \%$ femoral head coverage (FHC), and others use the distance measured between the femoral head and the shadow of ischium bone (HID) to determine treatment, special attention was directed toward evaluation of persistence of dysplasia in regard to these parameters (Table 4). We did not find any statistically significant difference in samples or in subgroups for these measurements. These measurements were statistically significant but on a population basis. Due to the small numerical values, they are not useful on a single-case basis.

\section{Discussion}

Variability in expert-opinion recommendations for the care of infant hip instability motivated this retrospective cohort case-only study on prospectively collected data and blinded data analysis [2-17]. Prior to the development of dynamic sonography described by Harcke, hip instability in the infant could not be precisely assessed $[4,5]$. Therefore, without natural history data and precise determinations of hip instability, the decision for treatment is based on the "expert opinion." Barlow stated that $88 \%$ of the unstable hips resolved in 2 months and proposed that the remainder be treated by his malleable splint [18]. Clarke and Castaneda recommended braces for hip instability that persists greater than 6-weeks [7]. Imrie et al. presented a population of 266 breech babies 
Table 1 Potential quantitative sonographic measurement predictors of early or late acetabular index normalization

\begin{tabular}{|c|c|c|c|c|c|c|c|c|c|}
\hline \multirow[t]{2}{*}{ Variable } & \multirow[t]{2}{*}{ Prevalence OR } & \multirow[t]{2}{*}{$95 \% \mathrm{Cl}$} & \multirow[t]{2}{*}{$p$} & \multicolumn{3}{|c|}{$\begin{array}{l}\text { Normalized before } 7 \text { months of age } \\
\text { (group I), number of hips }=17\end{array}$} & \multicolumn{3}{|c|}{$\begin{array}{l}\text { Normalized after } 7 \text { months of age } \\
\text { (group II), number of hips }=31\end{array}$} \\
\hline & & & & Median $(\mathrm{mm})$ & IQR (mm) & Min-max (mm) & Median (mm) & IQR (mm) & $\operatorname{Min}-\max (\mathrm{mm})$ \\
\hline LSCFaddMAD & 0.355 & $0.15-0.823$ & 0.016 & 4.35 & 1.7 & $3-7.2$ & 3.8 & 1 & $2.3-5.6$ \\
\hline LSCNmid & 31.15 & $1.74-558.69$ & 0.020 & 1.15 & 0.9 & $0.1-1.7$ & 1.75 & 0.55 & $1.1-2.4$ \\
\hline LSCFmad & 0.36 & $0.13-1.00$ & 0.050 & 3.75 & 1.2 & $1.8-5.5$ & 3.4 & 0.80 & $1.9-4.1$ \\
\hline LSCFaddBC & 12113.82 & $0.70-2.09$ & 0.059 & 0.57 & 0.1 & $0.42-0.64$ & 0.63 & 0.11 & $0.49-0.73$ \\
\hline LSCNmad & 0.58 & $0.32-1.07$ & 0.082 & 3.8 & 0.80 & $2.5-6.5$ & 3.3 & 1.9 & $-0.1-6.3$ \\
\hline FSCF $\Delta$ sce & 0.92 & $0.85-1.01$ & 0.092 & 2 & 10 & $-11-14$ & -2 & 9 & $-28-12$ \\
\hline $\mathrm{FSCNa}$ & 1.00 & $088-1.14$ & 0.966 & 59 & 5 & $55-68$ & 59 & 6 & $49-69$ \\
\hline LSCNa & 0.96 & $0.86-1.08$ & 0.526 & 64 & 7 & $51-79$ & 65 & 6 & $56-72$ \\
\hline $\mathrm{FSCNbc}$ & 1.06 & $098-1.14$ & 0.136 & 54.75 & 10.02 & $36.77-70.42$ & 58.68 & 12.81 & $43.24-90.68$ \\
\hline LSCNbc & 8.72 & $0.01-16345.16$ & 0.573 & 61.90 & 22.89 & $50.35-78.28$ & 65.89 & 13.11 & $54.49-85.71$ \\
\hline
\end{tabular}

Variable key: Time of measurement description ( $F S=$ first sonogram, $L S=$ last sonogram) and code for measurement (see Additional file 1 ). OR $=$ odds ratio, $\mathrm{Cl}=$ confidence interval. All infants' hips exhibited hip instability with stress and seated at rest with or without acetabular dysplasia on sonography. Normalization is radiographic $\mathrm{Al} \leq 25^{\circ}$

in which 193 had normal sonograms at 6 weeks of age; however, radiographs of these infants at 6 months of age showed 39 hips (29\% infants) with dysplasia "requiring treatment" with an orthosis [11]. Rosendahl et al. [16] followed 3613 patients in which 123 infants were treated based on clinical or sonography examination. Infants with minor dysplasia were treated only "if they were sonographically dislocated/dislocatable or borderline unstable" [16]. Infants with risk factors for DDH (breech presentation, "close family history") had a radiograph of the hips performed at 4 to 5 months of age [16]. The Guideline of the American Academy of Pediatrics summarized 118 studies from a larger set of 624 articles and presented no specific directions for managing instability without subluxation or dislocation [13]. Kohler et al. stated that persistent hip dysplasia in radiographs at 3.5 months of age and with limitation of abduction may justify the orthotic treatment to accelerate the acetabulum development; however, every case should be considered individually [12]. Graf [10] proposed that "the gliding movement of the femoral head" is acceptable as long as the bony acetabular roof is adequate or good. However, a deficient roof (Graf type IIc and worse) may damage the hip joint if the treatment is implemented [17]. Gans et al. suggested bracing for residual acetabular dysplasia in infantile DDH if the acetabular index is $\geq 30^{\circ}$ by 6 months of age [9]. Our natural history study challenges these traditions in that normalization of the AI occurred without treatment by age 7 months in 35\%, 12 in $67 \%, 18$ in $75 \%, 24$ in $81 \%$, and 36 in $100 \%$ of our hips. Shenton's line was intact and the $\mathrm{CE}$ angle was normal on all final follow-up radiographs.

We observed two variations in ossification of the acetabulum in unstable infant hips. In group I, normalization

Table 2 Sonographic measurement differences between acetabular index normalization time groups

\begin{tabular}{|c|c|c|c|c|c|c|c|}
\hline \multirow{4}{*}{$\begin{array}{l}\text { Sonographic } \\
\text { measurement }\end{array}$} & \multirow{2}{*}{\multicolumn{3}{|c|}{$\begin{array}{c}\text { Group I } \\
\begin{array}{c}\text { Normalized before } 7 \text { months of age, } \\
\text { number of hips }=17\end{array}\end{array}$}} & \multicolumn{3}{|c|}{ Group II } & \multirow[t]{4}{*}{$p$ value } \\
\hline & & & & $\begin{array}{r}\text { Normalized after } \\
\text { number of }\end{array}$ & $\begin{array}{l}\text { months of ag } \\
\text { ips }=31\end{array}$ & & \\
\hline & \multirow{2}{*}{$\begin{array}{c}\text { Number of hips measured } \\
\text { on sonography } \\
n / 17\end{array}$} & \multicolumn{2}{|c|}{$\begin{array}{l}\text { Sonographic } \\
\text { measurement }\end{array}$} & \multirow{2}{*}{$\begin{array}{c}\text { Number of hips measured } \\
\text { on sonography } \\
n / 31\end{array}$} & \multicolumn{2}{|c|}{$\begin{array}{c}\text { Sonographic } \\
\text { measurement }\end{array}$} & \\
\hline & & Mean (mm) & $\overline{\mathrm{SD}(\mathrm{mm})}$ & & Mean (mm) & $\overline{\mathrm{SD}(\mathrm{mm})}$ & \\
\hline LSCNmid & 10 & 0.99 & 0.58 & 16 & 1.7 & 0.42 & 0.001 \\
\hline LSCFaddMAD & 14 & 4.66 & 1.16 & 26 & 3.72 & 0.84 & 0.005 \\
\hline LSCFmad & 14 & 3.94 & 1.04 & 26 & 3.39 & 0.55 & 0.035 \\
\hline LSCFaddBC & 14 & 0.56 & 0.07 & 26 & 0.61 & 0.07 & 0.049 \\
\hline $\mathrm{FSTF} \Delta \mathrm{a} / \Delta \mathrm{D}$ & 17 & 3.25 & 9.29 & 28 & -0.68 & 5.38 & 0.078 \\
\hline FSCF $\Delta$ sce & 16 & 1.43 & 7.78 & 25 & -3.44 & 9.02 & 0.083 \\
\hline LSTFmad & 14 & 3.81 & 0.95 & 26 & 3.30 & 0.90 & 0.099 \\
\hline
\end{tabular}

Variable key: Time of measurement description (FS = first sonogram, LS = last sonogram) and code for measurement (see Additional file 1). All infants' hips exhibited hip instability with stress and seated at rest with or without acetabular dysplasia on sonography. Normalization is radiographic $\mathrm{Al} \leq 25^{\circ}$. 
Table 3 Study group demographic details for individual hips

\begin{tabular}{|c|c|c|c|c|}
\hline \multirow[t]{2}{*}{ Hip variable } & \multicolumn{2}{|c|}{ Group I (normalized at 7 months of age) $n=17$} & \multicolumn{2}{|c|}{ Group II (normalized after 7 months of age) $n=31$} \\
\hline & number & $\%$ & number & $\%$ \\
\hline \multicolumn{5}{|l|}{ Sex } \\
\hline Girls & 12 & 25.0 & 27 & 56.3 \\
\hline Boys & 5 & 10.4 & 4 & 6.3 \\
\hline \multicolumn{5}{|l|}{ Torticolis } \\
\hline No & 14 & 29.2 & 25 & 52.0 \\
\hline present & 3 & 6.3 & 6 & 12.5 \\
\hline \multicolumn{5}{|l|}{ Birth position } \\
\hline Normal & 7 & 14.6 & 11 & 22.9 \\
\hline Breech & 7 & 14.6 & $20^{*}$ & 41.7 \\
\hline Transverse & 1 & 2.1 & 0 & 0.0 \\
\hline No data & 2 & 4.1 & 0 & 0.0 \\
\hline \multicolumn{5}{|c|}{ Type of delivery } \\
\hline Physiological & 5 & 10.4 & 5 & 10.4 \\
\hline C-section & 7 & 14.6 & $25^{*}$ & 52.1 \\
\hline No data & 5 & 10.4 & 1 & 2.1 \\
\hline
\end{tabular}

Differences in acetabular normalization time for groups of infant hip instability with stress and seated at rest with or without acetabular dysplasia on sonography. ${ }^{*} p<0.001$; all infants had hip instability under stress and seated at rest with or without acetabular dysplasia on sonography.

of the AI was $\leq 7$ months of age, which is within the expected time of ossification. In group II, normalization of the AI was delayed until after 7 months of age. The two groups were different statistically by clinical factors and sonography. The sonographic measurements of morphology (LSCNmid, LSCFmad, LSCNmad, FSCF $\Delta$ sce) yielded only one statistically significant value, LSCNmid, with the others being almost significant. Measurement of instability by sonography (LSCFaddMAD) was statistically significant in predicting late AI normalization. Unexpectedly, greater instability was related to early normalization. We cannot account for this because another measurement of instability (LSCFaddBC) was almost significant in the opposite direction: i.e., greater instability was a reflection of later normalization of the AI (Table 2). Measurement of sonographic images results in very small numbered values (around one millimeter) and consequently is likely to be influenced greatly by discreet change in the position of the probe during the sonography examination; differences in muscle tone of the infant; or changes in the position of the joint from neutral to flexion or neutral to adduction, which is part of the sonographic examination protocol. Despite the strengths of the study (sample size, consecutive patient sample, rigorous methodology), there is possibility of information bias as the result of measurement parameters. Measurements were done by a single individual and although intraobserver variability was not formally calculated, we do not think that our results are likely to be driven by information bias because we performed repeated measures and reliability checks on the variables. Therefore, when the values of sonographic measurement are very small and overlapping between groups and the 95\% confidence interval (CI) is unreasonably wide, implying imprecise measurement (Table 1), we do not consider these values as reliable for predicting the outcome of AI normalization.

The scope of our study cannot conclude whether or not normalization of the AI at age 3 years will lead to continued normalization until maturity. The repeated radiograph in adolescence of our "additional insightful case" showed an upward tilt of the outward portion of the acetabular rim. The lateral margin of the acetabulum is formed by the secondary ossification centers of the acetabulum, and an abnormality of this area appears to be present [6]. On the basis of our inference in this

Table 4 The sonographic measurement of the a angle, FHC of more than $50 \%$ and HID

\begin{tabular}{|c|c|c|c|c|c|c|c|c|c|c|c|c|}
\hline \multirow[t]{2}{*}{ Measurement } & \multicolumn{4}{|c|}{ Coronal neutral } & \multicolumn{4}{|c|}{ Coronal flexion } & \multicolumn{4}{|c|}{ Coronal flexion adduction } \\
\hline & n & & SD & range & $\mathrm{n}$ & & SD & range & n & & SD & range \\
\hline a angle $\left[^{\circ}\right]$ & 39 & $59.9^{*}$ & 4.9 & $49-69$ & 44 & $59.8^{*}$ & 4.5 & $49-70$ & 45 & $58^{*}$ & 5.1 & $47-72$ \\
\hline $50 \%$ FHC [\%] & 45 & $57.1^{\#}$ & & $36.8-90.7$ & 50 & $55.3^{*}$ & 11.4 & $26.1-75.9$ & 49 & $49.6^{*}$ & 10.6 & 28.3-77.3 \\
\hline $\mathrm{HID}[\mathrm{mm}]$ & & $5.2^{\#}$ & & $2.4-8.3$ & & $5.13^{\#}$ & 1.39 & $2.9-10.3$ & & $5.7^{\#}$ & & $3.4-8.6$ \\
\hline
\end{tabular}

${ }^{*}$ mean, ${ }^{*}$ median, 50\% FHC - 50\% femoral head coverage, HID - distance measured between the femoral head and the shadow of ischium bone. We did not find any statistically important difference in samples as well as in subgroups with regard to the a angle, FHC of more than $50 \%$ and HID. 
study, we suggest a third variation of acetabular ossification in DDH that is not visible in childhood but is manifest in adolescence as an upward tilt of the outer portion of the acetabular rim. This single case illustrates the dilemma orthopedists face with $\mathrm{DDH}$, in that a normal AI in childhood may not mean the hip will remain normal throughout life. A similar observation was recorded by Tucci et al. in a group of 61 cases with DDH treated with a Pavlik harness in which $17 \%$ had an upward tilt of the outward portion of the acetabulum roof in a mean follow-up age of 12 years [22].

\section{Conclusions}

In conclusion, our sample is the first natural history study of hips with instability with stress and reduced at rest (with or without acetabular dysplasia on sonography measured as percent of head coverage or acetabular bone inclination as with the Graf $\alpha$ angle). All our patients had a normalized AI by 3 years of age without treatment. Two patterns of growth of the acetabulum were established: group I with normal ossification, and group II with delayed ossification. This natural history study supports the supposition that treatment may be unnecessary in infant hips with instability with stress and reduced at rest (with or without acetabular dysplasia on sonography measured as percent of head coverage or acetabular bone inclination as with the Graf $\alpha$ angle). We propose three patterns of acetabular ossification in unstable hips of infants: (I) normal ossification, (II) delayed ossification with normalization of the AI radiographically by age 3 years, and (III) abnormality in the secondary centers of ossification of the acetabulum.

\section{Consent}

Following the Institutional Review Board rules, written informed consent from the patient's guardian/parent was not needed as long as the published data were deidentified and collected retrospectively without any influence on treatment.

\section{Additional file}

Additional file 1: The list of measured values.

\footnotetext{
Abbreviations

D: Diameter of femoral head; a: Depth of acetabulum; a/D: ratio "a" to "D" (1); Al: Acetabular index; CFadd: Sonography coronal flexion adducted image; bc: bony coverage of the acetabulum (1); CE: Center-edge angle of Wiberg; CFadd: Sonography coronal flexion adducted image; CFn: Sonography coronal flexion neutral image; Cl: Confidence interval; CN: Sonography coronal neutral image; D: Diameter of femoral head; DDH: Developmental dysplasia of the hip; FS: First sonogram; hid: Head ischium distance; HID: Head ischium distance; IQR: Interquartile range; LS: Last sonogram; MAD: Mid acetabular distance; MID: Ischium cartilage thickness; sce: Central edge angle (on sonography); SD: Standard deviation; TF: Transverse flexion; TFadd: Transverse flexion adduction; $a$ : $a$ angle; $\Delta a / \Delta D$ : Change in ratio of change of depth of the acetabulum to the change of femoral head diameter
}

between adduction and neutral measurement; $\Delta \mathrm{HID}$ : Change in head ischium distance between adduction and neutral measurement; $\triangle M A D$ : Change in mid acetabular distance between adduction and neutral measurement; $\triangle \mathrm{sCE}$ : Change in sonographic central edge angle measurement between adduction and neutral measurement; $\Delta \mathrm{a}$ : Change in $a$ angle measurement between adduction and neutral measurement.

\section{Competing interests}

The authors declare that they have no competing interests.

\section{Authors' contributions}

$\mathrm{BP}$ - prepared the literature background, wrote the manuscript, measured sonograms, participated in the design of the study, conceived, and participated in its design and coordination. HTH - participated in the design of the study, was supervising radiologist in the study, conceived, participated in its design and coordination, and helped to draft the manuscript. LH participated in the design of the study and performed the statistical analysis. JRB - participated in the design of the study, was treating surgeon, conceived of the study, participated in its design and coordination, helped to draft the manuscript. All authors read and approved the final manuscript.

\section{Acknowledgement}

We thank Medical Editor Michelle Stofa for editorial assistance and clarification of data.

\section{Author details}

${ }^{1}$ Department of Orthopedics and Pediatric Orthopedics, Medical University of Lodz, 75 Drewnowska str, 91-002 Lodz, Poland. ²Department of Medical Imaging, Nemours/Alfred I. duPont Hospital for Children, 1600 Rockland Rd, Wilmington, DE 19803, USA. ${ }^{3}$ Department of Epidemiology, Nemours/Alfred I. duPont Hospital for Children, 1600 Rockland Rd, 19803 Wilmington, DE, USA. ${ }^{4}$ Department of Orthopedics, Nemours/Alfred I. duPont Hospital for Children, 1600 Rockland Rd, Wilmington, DE 19803, USA.

Received: 20 March 2014 Accepted: 29 September 2014

Published: 28 October 2014

\section{References}

1. Dorland's Illustrated Medical Dictionary. 27th ed. Philadelphia: W.B. Saunders; 1988.

2. Graf R: The diagnosis of congenital hip-joint dislocation by the ultrasonic Compound treatment. Arch Orthop Trauma Surg 1980, 97:117-133.

3. Graf R: Classification of hip joint dysplasia by means of sonography. Arch Orthop Trauma Surg 1984, 102:248-255.

4. Harcke HT, Clarke NM, Lee MS, Borns PF, MacEwen GD: Examination of the infant hip with real-time ultrasonography. J Ultrasound Med 1984, 3:131-137

5. Harcke HT, Grissom LE: Performing dynamic sonography of the infant hip. AJR Am J Roentgenol 1990, 155:837-844.

6. Bowen JR, Kotzias-Neto A: Developmental Dysplasia of the hip. Brooklandville, MD: Data Trace Publishing Company; 2006

7. Clarke NM, Castaneda P: Strategies to improve nonoperative childhood management. Orthop Clin North Am 2012, 43:281-289.

8. Eastwood DM: Neonatal hip screening. Lancet 2003, 361:595-597.

9. Gans I, Flynn JM, Sankar WN: Abduction bracing for residual acetabular dysplasia in infantile DDH. J Pediatr Orthop 2013, 33:714-718.

10. Graf R: Hip sonography: 20 years' experience and results. Hip Int 2007 17(Suppl 5):S8-S14.

11. Imrie M, Scott V, Stearns $P$, Bastrom T, Mubarak SJ: Is ultrasound screening for DDH in babies born breech sufficient? J Child Orthop 2010, 4:3-8

12. Kohler R, Dohin B, Canterino I, Pouillaude JM: Screening of congenital dislocation of the hip in the newborn. A systematic and rigorous clinical examination. A restricted use of imaging. Arch Pediatr 2003, 10:913-926. French.

13. Lehmann HP, Hinton R, Morello P, Santoli J: Developmental dysplasia of the hip practice guideline: technical report. Committee on quality improvement, and subcommittee on developmental dysplasia of the Hip. Pediatrics 2000, 105:E57.

14. Roposch A, Wright JG: Increased diagnostic information and understanding disease: uncertainty in the diagnosis of developmental hip dysplasia. Radiology 2007, 242:355-359. 
15. Roposch A, Liu LQ, Offiah AC, Wedge JH: Functional outcomes in children with osteonecrosis secondary to treatment of developmental dysplasia of the hip. J Bone Joint Surg Am 2011, 93:e145.

16. Rosendahl K, Aslaksen A, Lie RT, Markestad T: Reliability of ultrasound in the early diagnosis of developmental dysplasia of the hip. Pediatr Radiol 1995, 25:219-224.

17. Sibinski M, Adamczyk E, Higgs ZC, Synder M: Hip joint development in children with type Ilb developmental dysplasia. Int Orthop 2012, 36:1243-1246.

18. Barlow TG: Early diagnosis and treatment of congenital dislocation of the hip. J Bone Joint Surg (Br) 1962, 44:292-301.

19. Ortolani M: Un segno poco noto e sua importanza per la diagnosi precoce di prelussazione congenita dell'anca. Pediatria 1937, 45:129-135.

20. Caffey J, Ames R, Silverman WA, Ryder CT, Hough G: Contradiction of the congenital dysplasia-predislocation hypothesis of congenital dislocation of the hip through a study of the normal variation in acetabular angles at successive periods in infancy. Pediatrics 1956, 17:632-641.

21. Tonnis D: Normal values of the hip joint for the evaluation of $X$-rays in children and adults. Clin Orthop Relat Res 1976, 119:39-47.

22. Tucci JJ, Kumar SJ, Guille JT, Rubbo ER: Late acetabular dysplasia following early successful Pavlik harness treatment of congenital dislocation of the hip. J Pediatr Orthop 1991, 11:502-505.

doi:10.1186/1471-2474-15-355

Cite this article as: Pruszczynski et al:: Natural history of hip instability in infants (without subluxation or dislocation): a three year follow-up. BMC Musculoskeletal Disorders 2014 15:355.

\section{Submit your next manuscript to BioMed Central and take full advantage of:}

- Convenient online submission

- Thorough peer review

- No space constraints or color figure charges

- Immediate publication on acceptance

- Inclusion in PubMed, CAS, Scopus and Google Scholar

- Research which is freely available for redistribution 